\title{
Measuring preferences for analgesic treatment for cancer pain: How do African-Americans and Whites perform on choice-based conjoint (CBC) analysis experiments?
}

Salimah H Meghani ${ }^{1 *}$, Jesse Chittams ${ }^{2}$, Alexandra L Hanlon ${ }^{2}$ and Joseph Curry ${ }^{3}$

\begin{abstract}
Background: Conjoint Analysis (CA) can serve as an important tool to study health disparities and unique factors underlying decision-making in diverse subgroups. However, methodological advancements are needed in exploiting this application of CA. We compared the internal and external predictive validity and inter-temporal stability of Choice-based-Conjoint (CBC) analysis between African-Americans and Whites in the clinical context of preferences for analgesic treatment for cancer pain.

Methods: We conducted a prospective study with repeated-measures at two time-points ( $\mathrm{T} 1=$ baseline; T2 = 3-months). African-Americans $(n=102)$; and Whites $(n=139)$ with cancer-related pain were recruited from outpatient oncology clinics in Philadelphia. Informed by pilot work, a computer-assisted CBC experiment was developed using 5 attributes of analgesic treatment: type of analgesic; expected pain relief; type of side-effects; severity of side-effects; and out-of-pocket cost. The design included 2 choice alternatives, 12 random tasks, 2 holdout tasks, and maximum of 6 levels per attribute. The internal and external predictive validity of CBC was estimated using Root Likelihood (RLH) and Mean Absolute Error (MAE), respectively. Inter-temporal stability was assessed using Cohen's kappa.
\end{abstract}

Results: Whites predominantly traded based on "pain relief" whereas African-Americans traded based on "type of side-effects". At both time-points, the internal validity (RLH) was slightly higher for Whites than for AfricanAmericans. The RLH for African-Americans improved at T2, possibly due to the learning effect. Lexicographic (dominant) behavior was observed in $29 \%$ of choice datasets; Whites were more likely than African-Americans to engage in a lexicographic behavior (60\% vs. 40\%). External validity (MAE) was slightly better for African-Americans than for Whites at both time-points (MAE: T1 $=3.04 \%$ for African-Americans and $4.02 \%$ for Whites; T2 $=8.04 \%$ for African-Americans; $10.24 \%$ for Whites). At T2, the MAE increased for both groups possibly reflecting an increase in the complexity of pain treatment decision-making based on expectations (T1) as opposed to reality (T2). The intertemporal stability was fair for CBC attributes between T1 and T2 (kappa $=0.28,95 \%$ Cl: 0.24-0.32) and was not predicted by demographics including race.

Conclusions: While we found slight group differences, overall the internal and external predictive validity of CBC was comparable between African-Americans and Whites. We discuss some areas to investigate and improve internal and external predictive validity of CBC experiments.

\footnotetext{
* Correspondence: meghanis@nursing.upenn.edu

${ }^{1}$ Department of Biobehavioral Health Sciences, NewCourtland Center for

Transitions \& Health, University of Pennsylvania School of Nursing, Claire M.

Fagin Hall, 418 Curie Boulevard, Room 337, 19104-4217 Philadelphia, PA, USA

Full list of author information is available at the end of the article
} 


\section{Background}

The healthcare and funding structures in the U.S. have recently placed an unprecedented emphasis on the role of patients' perspectives in healthcare outcomes [1]. These directions necessitate understanding of techniques that improve assessment of patient-reported outcomes including the important intermediary outcomes of preferences and decision-making.

Conjoint Analysis (CA) is a valuation technique grounded in random utility theory [2] and mathematical psychology [3] to understand what people value and what drives them to choose one set of alternatives over another when faced with competing choices [4]. There has been a rapid increase in the application of CA in the health related research over the past decade [5]. The main premise of CA is that individuals derive utility from the properties or characteristics of a good rather than the good itself [6]. Thus, the utility or desirability of any good (e.g. health services or treatment alternatives) can be described based on the value of its separate, yet, conjoined parts. These separate but conjoined parts are termed "attributes" each with multiple "levels" [7]. By asking individuals to make trade-offs between an important but limited number of attributes, a unique set of values ("preference weights" or "part-worth utilities") can be derived. These preference weights are results of modeling the underlying latent utility function such that a higher preference weight represents a higher value an individual assigns to that attribute [7]. The attributes can then be compared to one another to ascertain the "relative importance" or the percentage of total variance in preferences that each attribute explains.

The relative importance an individual associates with an attribute is also expected to vary based on an individual's background (e.g., demographics) or clinical factors (e.g., expectations or past experiences with treatments) [8]. As may be evident, CA can serve as an important clinical and research tool to understand racial and ethnic disparities and what unique factors may underlie decision-making in diverse patient groups. This application of CA is beginning to be exploited in health literature $[9,10]$, and no studies to our knowledge, have compared the predictive validity and temporal stability of CA techniques among diverse subgroups of minorities.

Cancer pain treatment decisions are preferencesensitive [11] and clinically important racial disparities have been reported in preferences and adherence to analgesics for cancer pain [12-17]. CA can offer an important avenue to understand heuristics underlying cancer pain treatment decisions. However, the use of CA techniques to understand clinical disparities in preferences and decision-making requires addressing methodological issues including validity of this method in diverse patient populations. In this paper, we present one empirical example of comparing validity of conjoint analysis in diverse subgroups. More specifically, we compare the internal and external predictive validity and inter-temporal stability of Choice-based Conjoint $(\mathrm{CBC})$ analysis between African-Americans and Whites in the clinical context of understanding their preferences for analgesic treatment for cancer pain.

\section{Methods}

This study was approved by the Institutional Review Board of the University of Pennsylvania. Informed consent was obtained from all participants. A 3-month prospective observational study was conducted with repeated measures at two time-points, i.e., at baseline (T1) and 3-months (T2). Patients were recruited from two outpatient medical oncology clinics within the University of Pennsylvania Health System. Inclusion was based on self-identified African-Americans and Whites, at least 18 years of age, diagnosed with solid tumors or multiple myeloma, with cancer-related pain, and at least one prescription of around-the-clock pain medication. A trained research assistant made home visits to gather data at $\mathrm{T} 1$ and $\mathrm{T} 2$ at a time convenient for the patients.

\section{Conjoint analysis methodology}

The CBC study was designed in consultation with Sawtooth Technologies, Inc. CBC is one of the methods within the expanding repertoire of conjoint analysis techniques. It uses a decompositional design to observe consumer choices based on how they react to a series of changes in attribute levels of a good. The main advantage of $\mathrm{CBC}$ is that it presents choice questions in fullprofile, i.e., all attributes are presented to the respondent at one time, allowing respondents to make trade-offs between attribute levels closely mimicking how real life decisions are made [7].

The International Society for Pharmacoeconomics and Outcomes Research (ISPOR)'s Good Research Practices for Conjoint Analysis Task Force has recently published guidelines for the application of conjoint analysis in health [5]. Designing a CBC study involves systematic steps $[18,19]$. Key design elements include: Selection of attributes and levels that define the profiles in conjoint analysis tasks; construction of tasks; experimental design; and statistical analysis [18].

\section{Attributes and levels}

In our study, the construct of interest was preferences for analgesic treatment for cancer pain. Our interest was not to identify preferences for specific analgesics but most salient considerations patients have in using analgesia for cancer pain. Two constraints guided the identification of attributes: first, inclusion of the most salient attributes to minimize respondent burden and second, operationalization of attribute levels that are plausible. 
Both literature review and qualitative groundwork can serve to identify relevant attributes and levels [19]. In our study, separate qualitative focus groups with African-Americans and Whites [20] suggested six attributes that mattered the most to patients in considering analgesics for cancer pain treatment: 1) type of analgesic, 2) percent pain relief with analgesics, 3) type of sideeffects, 4) severity of side-effects, 5) out-of-pocket cost) and 6) analgesic-related beliefs.

Of the 6 identified attributes, 5 allowed operationalization into concrete levels (i.e., type of analgesic; degree of pain relief with analgesics; type of side-effects; severity of sideeffects; and out-of-pocket cost). The attribute of "analgesicrelated beliefs" was excluded because this attribute is endogenous to the respondent, and cannot be varied [5]. For instance, defining beliefs as "presence or absence of beliefs" or "strong" or "weak" beliefs was not meaningful. Thus, the research team, in consultation with Sawtooth Technologies, decided to study analgesic beliefs using a different discrete choice, trade-off technique, Maximum Difference Scaling (MaxDiff) analysis. MaxDiff is a paired comparison in which respondents are asked to choose from a given set of beliefs those that are "most" and "least" important in deriving patients' analgesic use [21]. Choices are varied systematically to understand which beliefs may underlie patients' decision-making to use analgesia (findings are presented as part of a separate paper in review).

\section{Construction of tasks}

Based on the final set of attributes, a computer-assisted, CBC experiment was developed. Efficient randomized design algorithms were used in the creation of choice profiles to yield unbiased estimates of participants' preferences. A heuristic optimization algorithm was applied using a balanced factorial design that has near perfect orthogonality (principle of independence, i.e., by varying variables individually, one can predict the combined effect of varying them jointly). This design was blocked into groups of 14 CBC tasks, with 2 treatment alternatives per task that were unique for each respondent. This study used CBC's Complete Enumeration task generation method, which forces alternatives within each task to be kept as different as possible (minimal overlap). The computer-assisted design was flexible, efficient, and robust to response ordering effects [22,23]. The randomized design permitted data to be aggregated question-byquestion allowing an examination of how utilities changed as respondents progressed through the interview. Further, the algorithms minimized the response burden, while yielding preference weights that have the smallest standard errors for the calculated sample size and design complexity.

The survey was field-tested with two separate groups of African-Americans and Whites with cancer-related pain $(\mathrm{N}=13$; African-Americans = 7, Whites =6) to determine the comprehension and ease of completion of computer-based CBC exercise. Of note, we included patients based on a range of computer literacy ('extremely comfortable' to 'not comfortable at all'). The investigator and a trained research assistant were available to assist patients with limited computer literacy. The final survey was modified based participants' suggestions for improving the instructions.

\section{Experimental design}

The T1 and T2 CBC designs were identical. Each design consisted of 12 random tasks and 2 holdout tasks ( $^{\text {th }}$ and $10^{\text {th }}$ tasks), for a total of 14 tasks. Two treatment alternatives were displayed per task. The holdout tasks were constructed so there would be a clearly preferred, but not overly dominant alternative in each task.

The final sample size was based on concepts of power calculation that are unique to $\mathrm{CBC}$ design. The conventional power calculations are not applicable in $\mathrm{CBC}$ studies; rather, conjoint analysis experts either apply rules of thumb or past experience in determining an appropriate sample size [24]. In our study, the final sample size was based on the past experience; we use the expected standard error of 0.05 recommended by Sawtooth Software, so our results could be compared more easily with the results of other studies that use CBC. The sample requirements were based on the number of attributes, the maximum number of levels per attribute, and the effects to be measured. A larger number of attributes provide more information on trade-offs but also encourage participants to simplify heuristics due to increased task complexity [24]. Similarly, more levels per attribute provide increased "preference granularity", but also increase the need for sample size to allow estimation of additional parameters [24].

The sample size was generated using a computer simulation that used random dummy respondent data for the specified $\mathrm{CBC}$ design using aggregate logit for estimating utilities. Also taken into consideration is the ability of respondents to reliably answer 10 to $20 \mathrm{CBC}$ questions [23,25]. The sample size and the number of questions were varied systematically until the expected standard error associated with the utility estimates was 0.05 or smaller. This yielded an estimate of 200 subjects. The model estimated was based on main effects. Further, we enrolled a total of 240 patients to account for a projected attrition rate of $20 \%$ due to advanced disease, death, and withdrawal for other reasons. Our sample size $(n=200)$ exceeded the sample size requirement using Johnson's Rule of Thumb, which recommends a minimum sample size of 125 for 2 choice alternatives; 12 task repetitions; and maximum number of 6 levels for any one attribute [24]. 


\section{Statistical analysis}

All analyses were conducted using Sawtooth Software $\mathrm{CBC} / \mathrm{HB}$ system [26]. Hierarchical Bayes (HB), allowed estimation of individual-level utilities using choice data. $\mathrm{HB}$ borrows information from every respondent in the dataset to improve the accuracy and stability of each individual's preference weights [7]. The relative importance and preference weights of specific analgesic attributes in determining the overall utility was analyzed using a random utility model which allowed analysis of clustered data (e.g., repeated measurements from multiple responses obtained from the same individual in this study). The function to be estimated was of the form:

$$
\mathrm{V}_{\mathrm{i}}=\mathrm{X}_{\mathrm{i}} \beta+e+u
$$

Where, $V_{i}=$ overall utility or preference associated with analgesic treatment $\mathrm{i} ; \mathrm{X}_{\mathrm{i}}=$ row vector of attributelevel codes representing alternative $i, \beta=$ vector of part worth utilities, $e=$ error that accounts for the differences amongst observations (measurement error), and $u=$ error that accounts for differences amongst respondents. A utility function that monotonically increases indicates that as the level of an attribute increases so does the individual's preference associated with that attribute. The converse is true for a utility function that monotonically decreases. The estimated utilities indicate the relative impact of different attribute levels on pain treatment decisions. The greater the relative size of the utility the greater the impact of the different attribute levels in determining the overall utility value.

Methods for internal and external predictive validity of the CBC exercise Two holdout tasks that looked exactly like the $\mathrm{CBC}$ scenarios were embedded in $14 \mathrm{CBC}$ tasks (one early in the survey and one late in the survey). The holdout tasks are not used in the measurement of preference weights but they provide essential insights into the validity and stability and of $\mathrm{CBC}$ responses [27]. The internal and external predictive validity of the $\mathrm{CBC}$ tasks was estimated using Root Likelihood (RLH) and Mean Absolute Error (MAE), respectively.

The RLH measures the goodness of fit between the estimated utilities and the respondent's choice data. We calculated the likelihood of each respondent choosing as he/she did on each task, by applying a logit model using estimates of the respondent's utilities. To compute RLH we multiplied these likelihoods together and took $n^{\text {th }}$ root of the likelihood, where $n$ is the total number of choices the respondent made. RHL is therefore the geometric mean of the predicted probabilities. If there were $\mathrm{k}$ alternatives in each choice task and we had no information about a respondent's utilities, we would predict that each alternative would be chosen with probability $1 / k$, and the corresponding RLH would be $1 / k$. RLH would be one if the fit were perfect.

The MAE is an average of the absolute differences between the choice model predictions and the aggregate choices respondents made for two holdout tasks included in the choice exercise. The logit exponent of the model was tuned for each racial group so as to minimize the MAE for that group.

Methods for inter-temporal stability of the CBC exercise The inter-temporal stability of the CBC was assessed using utilities from baseline and 3-months. The scores from each of the two assessment points were ranked from highest to lowest in terms of the percentage of overall importance assigned by the CBC utilities. Using Cohen's kappa statistic, the agreement among scores across the two time-points was assessed overall and individually within each $\mathrm{CBC}$ attribute. We used Cohen's kappa as it is thought to be a more robust measure than the simple percent agreement calculation since kappa takes into account the agreement occurring by chance. Based on magnitude guidelines, kappa values of 0 indicates no agreement, $0-.20$ as slight, .21-.40 as fair, .41-.60 as moderate, .61-.80 as substantial, and .81-1 as almost perfect agreement [28]. The agreement among the repeated assessment was also evaluated via the Spearman correlation coefficient, and an exploratory analysis to identify subgroups with strong retest characteristics.

Further, we ran a number of analyses to determine whether a very strong preference at baseline remains consistently strong at 3-months. Strong preference at baseline was defined as a 'relative importance' ranking for an attribute of $50 \%$ or more. Thus, a zero or one was assigned to each of the five $\mathrm{CBC}$ attributes for each subject based on whether the strong preference definition was met. If an attribute with the strongest preference also ranked highest or second highest at T2, the event was classified as 'stable', otherwise it was classified as 'not stable'. Logistic regression was employed using the SAS [29] software package to test for statistically significant associations between having a strong preference and potential demographic and clinical variables. Summary statistics and cross-tabulations were generated to further assess associations that were significant at the 0.05 level.

Methods to identify predictors of inter-temporal stability We examined whether differences in intertemporal stability can be explained by baseline demographic (education, income, race, age, marital status, and computer literacy) and clinical variables (general health and pain levels) collected at T1. For these set of analyses, we considered both the "actual difference" and the "absolute 
value of the difference" in the relative importance scores of an attribute within a patient from $\mathrm{T} 1$ to $\mathrm{T} 2$ as outcome measure of inter-temporal stability. The actual change is the mathematical subtraction of $\mathrm{T} 2-\mathrm{T} 1$, where $\mathrm{T} 2$ is the $\mathrm{CBC}$ relative importance score at $\mathrm{T} 2$, and $\mathrm{T} 1$ is the $\mathrm{CBC}$ relative importance score at $\mathrm{T} 1$ (for example, if $\mathrm{T} 2=0.49$ and $\mathrm{T} 1=0.54$, then the actual change would be -0.05 , but the absolute change would be 0.05 ). The actual difference was generated to test for a directional association and the absolute value of the difference was computed to test whether any of the baseline variables predict the general variability in the inter-temporal stability over time. Thus, the T1 to T2 differences at or near zero indicated better stability. We then performed several analyses to test for significant associations among all $5 \mathrm{CBC}$ attributes and baseline demographic and clinical variables.

Methods to identify change in information set from T1 to T2 Economic theory assumes that preferences are stable although a person's information set may change over time. Thus, we examined several patient-specific characteristics (general health, pain levels, pain-related interference, analgesic beliefs, and social support) for evidence of a statistically significant change from $\mathrm{T} 1$ to T2. All of the variables considered were continuous or on a likert scale. To determine what changed within the patient between $\mathrm{T} 1$ and $\mathrm{T} 2$ in terms of these factors, a paired T-test, or the Wilcoxon sign rank test was used depending on the symmetry of the distribution of the difference measure (T2 minus T1). If significant changes were detected, those changes were correlated with the changes in the relative importance scores of the $5 \mathrm{CBC}$ attributes from T1 to T2, using the Pearson and Spearman correlation coefficient.

Methods to identify systematic differences in those who completed both surveys and those who completed T1 only A comprehensive set of analyses was conducted to examine whether there were any systematic differences in the baseline characteristics between those who completed both surveys and those who only completed the survey at $T 1$. We began by performing a preliminary analysis of all outcomes (the $5 \mathrm{CBC}$ attributes) and baseline demographic variables and tested for differences in baseline measures between the group who only provided $\mathrm{T} 1$ data and those who provided both $\mathrm{T} 1$ and T2 data. The test for differential dropout was employed to see if the baseline variables were equally distributed between the two groups. These baseline comparisons were based on T-tests or Wilcoxon rank sum tests for continuous variables, depending on the symmetry of the distributions and on Chi-square or Fisher's Exact test depending on the cell sizes for binary or ordinal variables.

\section{Results}

A total of 241 patients (African-Americans =102; Whites $=139$ ) completed the CBC survey at baseline. There were significant differences between African-Americans and Whites on a number of demographic variables including gender, marital status, education, income, and insurance status (Table 1). The attrition rate from $\mathrm{T} 1$ to $\mathrm{T} 2$ was $17 \%(\mathrm{~N}=201)$. There was no evidence of differential dropout by key variables such as race $(\mathrm{p}=0.496)$ and general health status $(\mathrm{p}=0.612)$. The relative importance of the CBC choice structure remained stable from $\mathrm{T} 1$ to T2 (Table 2). The most important analgesic preference attributes at both time-points in descending order were 1) percent pain relief with analgesics, 2) type of analgesic side-effects 3) severity of side-effects 4) type of analgesics, and 5) out-of-pocket cost. At both time-points, Whites overwhelmingly traded based on "degree of pain relief" whereas for African-Americans, the most important factors in pain treatment were "type of analgesic side-effects" and "degree of pain relief" with analgesics (Table 3).

Subsequent analyses revealed that Whites had 2.8 times the odds of ranking strongest on the "percent pain relief with analgesics " attribute than African-Americans (95\% CI, 1.4 - 5.5; $\mathrm{p}=0.002)$. Those with higher income tended to be more likely to rank the "percent pain relief with analgesics" CBC attribute highest at T1 ( $\mathrm{p}=0.009)$. Lastly, those with higher education levels also tended to be more likely to rank the "percent pain relief with analgesics" attribute highest at T1 $(\mathrm{p}=0.007)$.

\section{Comparative validity}

Overall, the internal and external predictive validity of the $\mathrm{CBC}$ was comparable between African-Americans and Whites. At both time-points, the internal predictive validity $(\mathrm{RLH})$ of the $\mathrm{CBC}$ was slightly higher for Whites than for African-Americans (Table 4). The internal predictive validity for African-Americans improved at T2 nearly catching up to Whites possibly relating to learning effect (Table 4).

If we define dominant or lexicographic behavior in the data as having an RLH value greater than .750 (1.5 times chance) and a single attribute importance greater than $50 \%$, then this type of behavior was observed in $29 \%$ of the choice data sets. Of these data sets, $60 \%$ were for Whites and 40\% were for African-Americans.

As for external predictive validity (MAE), the CBC model was slightly better at predicting African-Americans' choices than for Whites (MAE $=3.04 \%$ for African-Americans and $4.02 \%$ for Whites). At T2, the MAE increased for both groups i.e., 8.04\% for African-Americans; $10.24 \%$ for Whites (MAE of 0 represents perfect agreement between the model and the aggregate choice data). The external validity 
Table 1 Demographic characteristics of study participants by race $(N=241)$

\begin{tabular}{|c|c|c|c|c|}
\hline Variable & Total $(\mathrm{N}=241)$ & African-Americans $(\mathrm{N}=102)$ & Whites $(\mathrm{N}=139)$ & p-values $\dagger$ \\
\hline \multicolumn{5}{|l|}{ Mean (SD) } \\
\hline Age & $53.7(11.0)$ & $52.7(10.1)$ & $54.5(11.6)$ & .194 \\
\hline Heath literacy score & $13.0(2.6)$ & $13.0(3.0)$ & $13.0(2.4)$ & .934 \\
\hline Comfort with computers & $3.3(1.4)$ & $3.1(1.4)$ & $3.5(1.4)$ & .033 \\
\hline \multicolumn{5}{|l|}{ Frequency (\%) } \\
\hline Gender & & & & .019 \\
\hline Male & $111(46)$ & $38(37)$ & $73(53)$ & \\
\hline Female & $130(54)$ & $64(63)$ & $66(47)$ & \\
\hline Marital status & & & & $<.001$ \\
\hline Married & $133(55)$ & $33(32)$ & $100(72)$ & \\
\hline Separated/ divorced/widowed & $62(26)$ & $42(41)$ & $20(14)$ & \\
\hline Never married & $46(19)$ & $27(27)$ & $19(14)$ & \\
\hline Education & & & & .011 \\
\hline Elementary & $3(1)$ & $2(2)$ & $1(2)$ & \\
\hline High school & $84(35)$ & $42(41)$ & $42(42)$ & \\
\hline College/trade school & $117(49)$ & $51(50)$ & $66(51)$ & \\
\hline More than college & $37(15)$ & $7(7)$ & $30(7)$ & \\
\hline Income & & & & $<.001$ \\
\hline$<30,000$ & $85(35)$ & $57(56)$ & $28(20)$ & \\
\hline $30-50,000$ & $44(18)$ & $26(25)$ & $18(13)$ & \\
\hline $50-70,000$ & $41(17)$ & $13(13)$ & $28(20)$ & \\
\hline $70-90,000$ & $25(11)$ & $3(3)$ & $22(16)$ & \\
\hline$>90,000$ & $46(19)$ & $3(3)$ & $43(31)$ & \\
\hline Health insurance & & & & $<.001$ \\
\hline Private & $123(51)$ & $30(29)$ & $93(67)$ & \\
\hline Medicaid & $33(14)$ & $28(27)$ & $5(4)$ & \\
\hline Medicare & $50(21)$ & $25(25)$ & $25(18)$ & \\
\hline Multiple & $26(11)$ & $13(13)$ & $13(9)$ & \\
\hline Other & $8(3)$ & $6(6)$ & $2(1)$ & \\
\hline Cancer type & & & & .895 \\
\hline Lung & $39(16)$ & $14(14)$ & $25(18)$ & \\
\hline Breast & $40(17)$ & $19(19)$ & $21(15)$ & \\
\hline Gl & $41(17)$ & $15(15)$ & $26(19)$ & \\
\hline GU/reproductive & $28(11)$ & $12(12)$ & $16(11)$ & \\
\hline Multiple myeloma & $36(15)$ & $17(16)$ & $19(14)$ & \\
\hline Other solid tumors & $57(24)$ & $25(24)$ & $32(23)$ & \\
\hline
\end{tabular}

$\mathrm{tp}$-values are based on t-tests for continuous variables and chi-squared for categorical variables.

of the T2 exercise again showed the model being slightly better at predicting African-Americans' choices (Table 4).

\section{Test for scale heterogeneity}

Aggregate scale factors were estimated for each subgroup in the process of minimizing the MAEs ("tuning the choice model"). At T1, scale factors were larger for AfricanAmericans (2.5) than for Whites (0.4), and nearly the reverse at T2 (African-Americans $=0.3$; Whites $=2.5$ ). This indicates that on average, Whites' choices became less random from T1 to T2, whereas African-Americans' choices became more random. This suggests that, to the extent the differences between scale factors within and between $\mathrm{T} 1$ and $\mathrm{T} 2$ are significant, experience with pain treatment may have clarified the choices for Whites, but made them more difficult for African-Americans. 
Table 2 Aggregate results of analgesic treatment utilities over-time estimated using CBC

\begin{tabular}{|c|c|c|c|c|c|}
\hline \multirow[b]{2}{*}{ Treatment attributes* } & \multirow[b]{2}{*}{ Levels } & \multicolumn{2}{|c|}{ At baseline $(\mathrm{N}=241)$} & \multicolumn{2}{|c|}{ At 3-months $(\mathrm{N}=201)$} \\
\hline & & $\begin{array}{c}\text { ‡Relative } \\
\text { importance }\end{array}$ & $\begin{array}{l}\text { tPreference } \\
\text { weights }\end{array}$ & $\begin{array}{c}\text { ‡Relative } \\
\text { importance }\end{array}$ & $\begin{array}{c}\text { tPreference } \\
\text { weights }\end{array}$ \\
\hline \multirow{5}{*}{$\begin{array}{l}\% \text { Pain relief with } \\
\text { analgesics }\end{array}$} & & 32.53 & & 32.11 & \\
\hline & $<50 \%$ & & -90.3 & & -91.8 \\
\hline & $60-70 \%$ & & -12.9 & & -13.8 \\
\hline & $80-90 \%$ & & 30.9 & & 36.9 \\
\hline & $100 \%$ & & 72.3 & & 68.7 \\
\hline \multirow{6}{*}{$\begin{array}{c}\text { Type of analgesic side- } \\
\text { effects }\end{array}$} & & 23.28 & & 19.71 & \\
\hline & Confusion & & -37.8 & & -23.4 \\
\hline & Drowsiness/Dizziness & & 23.8 & & 12.0 \\
\hline & Heartburn/Sour Stomach & & 13.7 & & 9.2 \\
\hline & Constipation & & 16.9 & & 19.1 \\
\hline & Nausea/Nomiting & & -16.7 & & -16.9 \\
\hline \multirow[t]{4}{*}{ Severity of side-effects } & & 17.81 & & 17.16 & \\
\hline & Mild & & 32.5 & & 34.1 \\
\hline & Moderate & & 24.1 & & 17.6 \\
\hline & Severe & & -56.6 & & -51.7 \\
\hline \multirow[t]{3}{*}{ Type of analgesic } & & 14.85 & & 15.86 & \\
\hline & $\begin{array}{l}\text { Oxycontin or Morphine- } \\
\text { like }\end{array}$ & & 19.5 & & 19.2 \\
\hline & Motrin or Aleve-like & & -19.5 & & -19.2 \\
\hline \multirow[t]{6}{*}{ Out-of-pocket cost } & & 11.53 & & 15.17 & \\
\hline & $\$ 10-\$ 20$ & & 24.5 & & 28.7 \\
\hline & $\$ 30-\$ 40$ & & 20.8 & & 18.3 \\
\hline & $\$ 50-\$ 60$ & & -0.16 & & 6.5 \\
\hline & $\$ 70-\$ 90$ & & -11.9 & & -6.4 \\
\hline & $\$ 100$ or more & & -33.1 & & -47.1 \\
\hline
\end{tabular}

*The attributes were identified based on pilot work with African Americans and Whites with cancer pain. ¥Relative importance scores sum to 100 across all attributes; †Aggregate utilities associated with each level of the attribute; smaller or more negative preference weight indicates less preference for that level of an attribute.

\section{Inter-temporal stability}

Inter-temporal stability was measured for each participant who had data available at both time-points. The Inter-temporal stability of CBC utilities over the 3month time period yielded a Kappa of 0.28 with a $95 \%$ confidence interval of 0.24 to 0.32 . The correlation between $\mathrm{T} 1$ and $\mathrm{T} 2$ rankings as measured by the Spearman correlation coefficient was consistent with the Kappa (Spearman Coefficient $=0.37$ ). While in general there were differences between the T1 and T2 CBC utilities, there were subgroups that showed consistency from T1 to T2. Specifically, $88 \%$ of those who ranked the attribute of "percent pain relief" highest (defined as relative importance score of at least 50\%) at $\mathrm{T} 1$, also ranked the "percent pain relief" high $\left(1^{\text {st }}\right.$ or $\left.2^{\text {nd }}\right)$ during $\mathrm{T} 2 ; 77 \%$ of those who ranked the "analgesic side-effects" attribute highest during $\mathrm{T} 1$, also ranked this attribute high $\left(1^{\text {st }}\right.$ or $2^{\text {nd }}$ ) during $\mathrm{T} 2$; and $71 \%$ of those who ranked the "type of analgesic" attribute highest during $\mathrm{T} 1$, also ranked the "type of analgesic" high $\left(1^{\text {st }}\right.$ or $\left.2^{\text {nd }}\right)$ during T2 (Table 5$)$.

\section{Predictors of inter-temporal stability}

We found no statistically significant associations (at the alpha $=0.05$ level) between differences in inter-temporal stability and any of the baseline (education, income, race, age, marital status, and computer literacy) and clinical variables (general health and pain levels). The Pearson and Spearman correlation coefficients ranged between -0.2 and 0.2 for all of the continuous baseline predictor variables. This was the case, regardless of whether intertemporal stability was measured as the actual change or the absolute change from T1 to T2. 
Table 3 Aggregate utilities of analgesic treatment for cancer pain "by race" over-time

\begin{tabular}{|c|c|c|c|c|c|c|}
\hline \multirow[b]{2}{*}{ Attributes \& levels $†$} & \multicolumn{3}{|c|}{ At baseline $(N=241)$} & \multicolumn{3}{|c|}{ At 3-months $(\mathrm{N}=201)$} \\
\hline & $\begin{array}{l}\text { Whites } \\
(N=139)\end{array}$ & $\begin{array}{c}\text { African-Americans } \\
(\mathrm{N}=102)\end{array}$ & $\overline{p \text {-values }}$ & $\begin{array}{l}\text { Whites } \\
(N=114)\end{array}$ & $\begin{array}{l}\text { African-Americans } \\
(\mathrm{N}=87)\end{array}$ & $\overline{p \text {-values }}$ \\
\hline \% Pain relief with analgesics & $36.71^{\ddagger}$ & $26.83^{\neq}$ & $<0.001$ & $35.65^{\neq}$ & $27.45^{\ddagger}$ & .006 \\
\hline$<50 \%$ & -102.5 & -73.8 & .000 & -101.7 & -78.8 & .006 \\
\hline $60-70 \%$ & -15.7 & -9.12 & .002 & -15.2 & -11.9 & .337 \\
\hline $80-90 \%$ & 37.1 & 22.6 & .000 & 40.4 & 32.3 & .062 \\
\hline $100 \%$ & 81.1 & 60.3 & .000 & 76.5 & 58.5 & .007 \\
\hline Type of analgesic side-effects & $19.29^{\ddagger}$ & $28.72^{\ddagger}$ & $<0.001$ & $16.96^{\ddagger}$ & $23.30^{\ddagger}$ & .009 \\
\hline Confusion & -32.1 & -45.5 & .081 & -11.7 & -38.8 & .000 \\
\hline Drowsiness/dizziness & 21.0 & 27.6 & .145 & 12.8 & 11.1 & .717 \\
\hline Heartburn/sour stomach & 13.3 & 14.4 & .865 & 7.8 & 11.0 & .518 \\
\hline Constipation & 18.9 & 14.2 & .448 & 13.5 & 26.5 & .047 \\
\hline Nausea/vomiting & -21.1 & -10.7 & .056 & -22.4 & -9.8 & .048 \\
\hline Severity of side-effects & $18.55^{\ddagger}$ & $16.81^{\ddagger}$ & .225 & $19.03^{\neq}$ & $14.71^{\neq}$ & .027 \\
\hline Mild & 33.0 & 31.8 & .636 & 38.2 & 28.7 & .022 \\
\hline Moderate & 26.7 & 20.6 & .010 & 18.7 & 16.1 & .212 \\
\hline Severe & -59.7 & -52.3 & .112 & -56.9 & -44.8 & .034 \\
\hline Type of analgesic & $13.52^{\ddagger}$ & $16.66^{\ddagger}$ & .176 & $14.83^{\ddagger}$ & $17.19^{\ddagger}$ & .413 \\
\hline Oxycontin or morphine-like pain medicine & 19.9 & 19.0 & .904 & 23.4 & 13.8 & .272 \\
\hline Motrin or aleve-like pain medicine & -19.9 & -19.0 & .904 & -23.4 & -13.8 & .272 \\
\hline Out of pocket cost & $11.93^{\ddagger}$ & $10.98^{\ddagger}$ & .355 & $13.51^{\ddagger}$ & $17.33^{\ddagger}$ & .017 \\
\hline$\$ 10-\$ 20$ & 25.0 & 23.8 & .608 & 24.7 & 33.9 & .003 \\
\hline$\$ 30-\$ 40$ & 22.0 & 19.1 & .122 & 16.1 & 21.2 & .019 \\
\hline$\$ 50-\$ 60$ & -.11 & -.23 & .889 & 7.9 & 4.6 & .023 \\
\hline$\$ 70-\$ 90$ & -12.3 & -11.5 & .598 & -5.9 & -7.1 & .466 \\
\hline$\$ 100$ or more & -34.6 & -31.1 & .232 & -42.8 & -52.7 & .037 \\
\hline
\end{tabular}

†Aggregate utilities associated with each level of the attribute; smaller or more negative preference weight indicates less preference for that level of an attribute. \#Relative importance scores sum to 100 across all attributes. P-values are based on 2-tailed two sample t-test.

\section{Change in information set from T1 to T2}

We identified a number of patient-specific variables that exhibited statistically significant change from T1 to T2. These included "physical health" in past 30 days $(\mathrm{p}=0.03)$; "worst pain" level $(\mathrm{p}=0.002)$ and pain-related "functional interference" $(\mathrm{p}=0.015)$ measured using Brief Pain Inventory [30]; and "pain management barriers" (harmful effects, $\mathrm{p}=0.048$; physiological effects, $\mathrm{p}=0.029$; and total number of pain related barriers, $\mathrm{p}=0.017$ ) measured using Barriers' Questionnaire [31]. Of note, while the above mentioned patient-specific variables changed significantly over the 3 month period, none of the changes were found to be correlated with changes in the patient preferences as measured by changes in the relative importance scores from $\mathrm{T} 1$ to $\mathrm{T} 2$. All of the Pearson and Spearman correlations were well within -0.2 and 0.2 .

\section{Systematic differences in those who completed both surveys} and those who completed T1 only

We found no statistically significant systematic differences in baseline demographics [age $(\mathrm{p}=0.9052)$, gender

Table 4 Internal and external predictive validity of CBC utilities by race over-time

\begin{tabular}{|c|c|c|c|c|c|c|}
\hline & \multicolumn{4}{|c|}{ Internal predictive validity } & \multicolumn{2}{|c|}{ External predictive validity } \\
\hline & tRLH (baseline) & $95 \% \mathrm{Cl}$ & tRLH (3-months) & $95 \% \mathrm{Cl}$ & ‡MAE (baseline) & ‡MAE (3 months) \\
\hline African-Americans & 0.789 & $0.767-0.812$ & 0.826 & $0.804-0.848$ & $3.04 \%$ & $8.04 \%$ \\
\hline Whites & 0.849 & $0.835-0.863$ & 0.846 & $0.828-0.865$ & $4.20 \%$ & $10.24 \%$ \\
\hline
\end{tabular}

†RLH = Root Liklihood; $¥ M A E=$ Mean Absolute Error. 
Table 5 Inter-temporal stability of CBC utilities over-time

\begin{tabular}{|c|c|c|c|c|c|c|c|}
\hline \multirow[b]{2}{*}{$\begin{array}{l}\text { Dominant } \\
\text { utility }^{*}\end{array}$} & \multicolumn{3}{|c|}{ Overall } & \multicolumn{2}{|c|}{ African-Americans } & \multicolumn{2}{|c|}{ Whites } \\
\hline & $\begin{array}{l}\text { Sensitivity/ } \\
\text { specificity }^{\dagger}\end{array}$ & $\begin{array}{l}\text { Kappa } \\
(95 \% \mathrm{Cl})\end{array}$ & $\begin{array}{c}\text { Odds ratio }^{\ddagger} \\
(95 \% \mathrm{Cl})\end{array}$ & $\begin{array}{l}\text { Sensitivity/ } \\
\text { specificity }^{\dagger}\end{array}$ & $\begin{array}{l}\text { Kappa }^{\ddagger} \\
(95 \% \mathrm{Cl})\end{array}$ & $\begin{array}{l}\text { Sensitivity/ } \\
\text { specificity }^{\dagger}\end{array}$ & $\begin{array}{c}\text { Kappa } \\
(95 \% \mathrm{Cl})\end{array}$ \\
\hline $\begin{array}{l}\% \text { Relief with } \\
\text { analgesics }\end{array}$ & $0.88 / 0.40$ & $0.17(0.08,0.25)$ & $4.6(1.9,11.6)$ & $0.62 / 0.46$ & $0.04(-0.10,0.17)$ & $0.97 / 0.34$ & $0.22(0.12,0.33)$ \\
\hline $\begin{array}{l}\text { Side-effects } \\
\text { type }\end{array}$ & $0.78 / 0.59$ & $0.13(0.04,0.22)$ & $5.0(1.6,15.9)$ & $0.86 / 0.58$ & $0.24(0.08,0.39)$ & $0.50 / 0.60$ & $0.02(-0.07,0.10)$ \\
\hline $\begin{array}{l}\text { Type of } \\
\text { analgesic }\end{array}$ & $0.71 / 0.80$ & $0.32(0.17,0.46)$ & $10.0(3.6,27.6)$ & $0.80 / 0.77$ & $0.33(0.13,0.54)$ & $0.64 / 0.83$ & $0.29(0.08,0.51)$ \\
\hline
\end{tabular}

$(p=0.0579)$, race $(p=0.5998)$, education $(p=0.9835)$, health literacy $(p=0.3317)$, income $(p=0.0522)$, type of insurance $(p=0.0631)$, social support $(p=.2157)]$ and clinical characteristics [general health $(p=0.9214)$, average pain levels $(p=0.7256)$, pain-related functional interference $(\mathrm{p}=.4318)$, and reported analgesic barriers $(p=0.7555)]$ between those who completed both surveys and those who completed the survey at T1 only.

\section{Discussion}

Widespread and concerning disparities have been documented in a variety of clinical outcomes in the U.S, although sources of disparities are not adequately explained. We continue to remain deficient in effective methods to understand sources of disparities. Conjoint Analysis can serve as an important tool to understand what unique factors may underlie decision-making of diverse subgroups, although methodological advancements are needed in exploiting this application of CA. In this study, we compared the predictive validity and temporal stability of $\mathrm{CBC}$ in eliciting preferences for cancer pain treatment between African-Americans and Whites.

Despite 3-month duration between the baseline and subsequent assessment, we found that the "overall" choice structures (relative importance and preference weights) remained stable from T1 to T2 (Table 2). Also, the most salient factors for pain treatment decisionmaking remained stable for both African-Americans and Whites (Table 3). The values of estimated utilities conformed to logic and a priori assumptions. For instance, for both groups, the estimated values became more negative as the attribute levels became less favorable, (e.g. more cost or less relief) (Table 3). Interestingly, for Whites expectation of "pain relief" was the more salient and consistent factor, whereas AfricanAmericans traded between "side-effects" and "pain relief" suggesting unique concerns underlying analgesic taking behavior and possibly disparate clinical management of pain and side-effects between the two groups.

\section{Internal predictive validity}

At both time-points, the internal predictive validity (RLH) of the CBC was slightly higher for Whites than for African-Americans (Table 4). This indicates that Whites were slightly more consistent than AfricanAmericans when expressing their choices as indicated by their higher RLH (Table 4). This may reflect a higher level of involvement by Whites in the pain treatment process prior to $\mathrm{T} 1$ (e.g., reading up on pain treatment alternatives, doctor consultations, or participation in cancer support groups, etc.). This also suggests that Whites used lexicographic decision rules more often than African-Americans when choosing pain treatments alternatives.

In our study, lexicographic behavior was observed in $29 \%$ of choice data sets. Within this group, Whites were more likely than African-Americans to engage in a lexicographic or dominant behavior (60\% vs. $40 \%)$. Lexicographic preferences occur when only one attribute matters to the individuals in considering a good or service resulting in unwillingness to trade more or less of one attribute in favor or detriment of the other. Our study provided evidence that on average Whites were significantly more likely to trade based on expected pain relief from analgesics than any other attribute (see Table 3). Lexicographic preferences may arise both from complexity of the conjoint choices but also an individual's past experiences or expectations [32].

While we found evidence of lexicographic decision behavior in the data, we cannot determine whether it reflects actual preferences or simplifying processes since we did not debrief patients about how they made their choices. However, the concern about lexicographic preferences arising from mental shortcuts is mitigated in studies where participants view the task as relevant to 
their condition and thus are highly motivated to answer the questions [33]. For instance, in our study all patients had cancer-related pain and they may view certain levels of attributes as most salient or a "must" in pain treatment decision-making.

The fact that internal predictive validity for AfricanAmericans and Whites became almost similar at T2 (Table 4) may indicate that the relative proportion of error in prediction for African-Americans at T1 may relate to learning effect (processing and evaluating the full-profile choice). Allowing participants to become familiar with $\mathrm{CBC}$ exercise, e.g. by incorporating mock $\mathrm{CBC}$ questions early on, may overcome learning bias and improve estimation of utilities.

\section{External predictive validity}

Based on our findings on the external validity, we found that it was easier to predict the pain treatment decisions for African-Americans than for Whites as indicated by the smaller MAE for African-Americans at both timepoints (Table 4). One likely explanation for this difference is that the two CBC holdout tasks used in determining the MAE might have presented alternative pain treatment scenarios that were more differentiated (with respect to preference system) for African-Americans than for Whites. Further, the MAE increased from T1 to T2 for both Whites and African-Americans possibly reflecting an increase in the complexity of making pain treatment decisions based on expectations (T1) as opposed to reality (T2).

\section{Inter-temporal stability}

Based on the magnitude guidelines for kappa values, we found evidence of fair inter-temporal stability $(0.28,95 \%$ confidence interval $=0.24$ to 0.32 ) for the $\mathrm{CBC}$ attributes over time. While the inter-temporal stability was only fair, this discrepancy is to be expected for a number of reasons: (1) we used Hierarchical Bayes (HB) estimation procedures in computing part-worth utilities or preference weights. HB estimates an individual's scores by using the individual's data and sharing data from other respondents [26]. Since the pool of respondents differed between T1 and T2 we would expect the individual scores to be different. This may specially affect stability of utilities for those with marginal preferences for an attribute. This is plausible since when we partitioned our analysis for only those with a high relative importance score of $50 \%$ or more on an attribute at $\mathrm{T} 1$, we found that a high percentage of individuals was likely to maintain their preferences at T2 (see Table 5); (2) one source of difference between time-points could be attributable to differences in the set of choice questions since a patient did not answer the same set of choice questions from the randomized block design at both time-points;
(3) conjoint studies can be cognitively challenging and learning effects are well known; based on our findings of internal predictive validity (RLH; Table 4), learning almost certainly occurred between $\mathrm{T} 1$ and $\mathrm{T} 2$ possibly resulting in observed differences in temporal stability; (4) from a conceptual perspective, preferences may evolve overtime as a result of change in information set; a lack of stability could be considered good if it captures an individual's real life experiences. For example, one might prefer pain relief going into treatment but prefer fewer side effects after experiencing treatment for three months. However, based on the analysis conducted, while we identified a number of relevant clinical variables that exhibited a statistically significant change from $\mathrm{T} 1$ to $\mathrm{T} 2$, none of the changes were found to be correlated with changes in the patient preferences from $\mathrm{T} 1$ to T2. Thus, at least based on the variables tested, change in information set could not explain the observed intertemporal stability from $\mathrm{T} 1$ to $\mathrm{T} 2$.

\section{Study limitations}

The findings of our study should be interpreted in the light of several limitations. In this paper, we presented a single empirical example of how predictive validity tests could be conducted as part of a CBC experiment. We estimated separate models to evaluate the performance of African-Americans and Whites on the predictive validity tests. However, estimating separate models is only one way to estimate systematic differences in preferences between groups. Alternative approaches include interaction models, nested models, and latent class models. Further, this study used CBC's Complete Enumeration task generation method, which forces alternatives within each task to be kept as different as possible (minimal overlap). That, together with showing only two alternatives per task, also increased the likelihood of patients using lexicographic-type decision rules. Further, our sample was limited to cancer patients from one health system. Findings may vary in patients in other contexts.

\section{Conclusions}

The analysis presented in this paper pertained to methodological issues; our goal was to assess how AfricanAmericans and Whites performed on a systematically designed $\mathrm{CBC}$ experiment. More specifically we sought to compare internal and external predictive validity and inter-temporal stability of $\mathrm{CBC}$ in these two groups. Based on the comparative validity findings, we conclude despite slight group differences, overall the internal and external predictive validity of $\mathrm{CBC}$ was comparable between African-Americans and Whites. For internal validity, we found that a learning bias may have operated more so for African-Americans. Allowing participants to become familiar with $\mathrm{CBC}$ exercise, e.g., by 
incorporating mock $\mathrm{CBC}$ questions early on, may improve estimation of utilities by overcoming learning bias. Dominant (or lexicographic) behavior was observed in a minority (29\%) of choice data sets. Incorporating debriefing or qualitative interviews as part of $\mathrm{CBC}$ exercise may provide insights into sources of dominant or lexicographic preferences. Unlike traditional instruments validated within the classical test theory paradigm, validity is not an inherent property of CBC surveys; holdout tasks may be included as part of $\mathrm{CBC}$ exercise as one way to study predictive validity. Validity of $\mathrm{CBC}$ survey is also based on pragmatic issues such as task complexity (e.g., number of attributes, number of levels per attribute, and number of tasks per respondent) and task relevance (e.g., how plausible attributes and levels are within a given context). These considerations should be taken into account in designing any rigorous CBC study, including studies to elucidate clinical disparities.

\section{Competing interests}

SHM, ALH and JChittams declare no financial or non-financial competing interests. JCurry has declared competing financial interest as the President of Sawtooth Technologies, Inc. The company routinely assists academics in the design and analysis of conjoint analysis studies. The authors declare that they have no competing interests.

\section{Authors' contributions}

This study was conceived by SHM. SHM contributed to all aspects of research and writing. JCurry assisted with the analysis of conjoint data as well as offered methodological input during all the stages of research. ALH and JChittams assisted with the analysis of the temporal stability aim of the study. Writing of the manuscript was led by SHM and all authors provided critical feedback and approved the final version.

\section{Acknowledgements}

This study was supported by the ARRA Challenge Grant to Dr. Salimah H. Meghani from the National Institutes of Health/NINR (NIHRC1NR011591).

\section{Author details}

'Department of Biobehavioral Health Sciences, NewCourtland Center for Transitions \& Health, University of Pennsylvania School of Nursing, Claire M. Fagin Hall, 418 Curie Boulevard, Room 337, 19104-4217 Philadelphia, PA, USA. ${ }^{2}$ University of Pennsylvania School of Nursing, 418 Curie Blvd. Room 492, 19104 Philadelphia, PA, USA. ${ }^{3}$ Sawtooth Technologies, Inc., 1500 Skokie Boulevard, 60062 Northbrook, IL, USA.

Received: 17 December 2012 Accepted: 10 October 2013

Published: 18 October 2013

\section{References}

1. Selby JV, Beal AC, Frank L: The patient-centered outcomes research institute (PCORI) national priorities for research and initial research agenda. JAMA 2012, 307(15):1583-1584.

2. Thurstone LL: A law of comparative judgment. Psychol Rev 1927, 34:278-286.

3. Luce D, Tukey J: Simultaneous conjoint measurement. J Math Psychol 1964, 1:1-27.

4. Green $P$, Rao V: Conjoint measurement for quantifying judgmental data. J Mark Res 1971, 8:355-363.

5. Bridges JF, et al: Conjoint analysis applications in health-a checklist: a report of the ISPOR good research practices for conjoint analysis task force. Value Health 2011, 14(4):403-413.

6. Lancaster JK: A new approach to consumer theory. J Polit Econ 1966, 74:132-157.

7. Orme BK: Getting started with conjoint analysis: Strategies for product design and pricing research. Madison: Research Publishers LLC; 2005.
8. Rosko MD, McKenna W: Modeling consumer choices of health plans: a comparison of two techniques. Soc Sci Med 1983, 17(7):421-429.

9. Constantinescu F, et al: Racial disparities in treatment preferences for rheumatoid arthritis. Med Care 2009, 47(3):350-355.

10. Hawley ST, et al: Preferences for colorectal cancer screening among racially/ ethnically diverse primary care patients. Med Care 2008, 46(9 Suppl 1):S10-S16.

11. Meghani SH, Keane A: Preference for analgesic treatment for cancer pain among African Americans. J Pain Symptom Manage 2007, 34(2):136-147.

12. Anderson $\mathrm{KO}$, Green $\mathrm{CR}$, Payne R: Racial and ethnic disparities in pain: causes and consequences of unequal care. J Pain 2009, 10(12):1187-1204.

13. Green $C R$, et al: The unequal burden of pain: confronting racial and ethnic disparities in pain. Pain Med 2003, 4(3):277-294.

14. Meghani $\mathrm{SH}$, et al: Advancing a national agenda to eliminate disparities in pain care: directions for health policy, education, practice, and research. Pain Med 2012, 13(1):5-28.

15. Shavers VL, Bakos A, Sheppard VB: Race, ethnicity, and pain among the U.S. adult population. J Health Care Poor Underserved 2010, 21(1):177-220.

16. Cintron A, Morrison RS: Pain and ethnicity in the United States: a systematic review. J Palliat Med 2006, 9(6):1454-473.

17. Meghani SH, Bruner DW: A pilot study to identify correlates of intentional versus unintentional nonadherence to analgesic treatment for cancer pain. Pain Manag Nurs 2013, 14(2):e22-30.

18. Johnson FR, et al: Constructing experimental designs for discrete-choice experiments: report of the ISPOR conjoint analysis experimental design good research practices task force. Value Health 2013, 16(1):3-13.

19. Ryan $M$, et al: Eliciting public preferences for healthcare: a systematic review of techniques. Health Technol Assess 2001, 5(5):1-186.

20. Meghani SH: Choice-based conjoint analysis (CBC) to elicit preferences for cancer pain treatment between and among African Americans and whites: a pilot study. J Pain 2010, 11(4):20.

21. Sawtooth Software Inc: The MaxDiff System: Technical Paper. Sequim: Sawtooth Software, Inc:: Sawtooth Software Technical Paper Series; 2008.

22. Ryan $\mathrm{M}$, Farrar S: Using conjoint analysis to elicit preferences for health care. BMJ 2000, 320(7248):1530-1533.

23. Sawtooth Software: Choice-based Conjoint (CBC) Technical Paper. Sequim: Sawtooth Software, Inc.; 2008.

24. Johnson FR, Yang J, Mohamed AF: In Defense of Imperfect Experimental Designs; Statistical Efficiency and Measurement Error in Choice-Format Conjoint Analysis, Sawtooth Software Conference Proceedings; 2012. http://www.sawtoothsoftware. com/download/techpap/2012Proceedings.pdf.

25. Johnson RM, Orme BK: How Many Questions Should You Ask in Choice-Based Conjoint Studies. Beaver Creek, Colorado: Paper presented at the American Marketing Association Advanced Research Techniques Forum; 1996. http://www.skimgroup.com/images/stories/technicalpapers/CBC-related\% 20papers/howmanyq.pdf.

26. Sawtooth Software Inc: The CBC/HB System for Hierarchical Bayes Estimation Version 5.0 Technical Paper. Sequim: Sawtooth Software, Inc:; 2009.

27. Orme BK, Alpert Ml, Christensen E: Assessing the validity of conjoint analysis. Sequim: Sawtooth Software, Inc.; 1997.

28. Landis JR, Koch GG: The measurement of observer agreement for categorical data. Biometrics 1977, 33(1):159-174.

29. SAS Institute Inc: SAS/STAT 9.2 User's Guide. Cary, NC: SAS Institute Inc Copyright $\odot$ 2008. Cary, NC, USA: SAS Institute Inc; 2008.

30. Cleeland CS, Ryan KM: Pain assessment: global use of the brief pain inventory. Ann Acad Med Singap 1994, 23(2):129-138.

31. Gunnarsdottir $S$, et al: Patient-related barriers to pain management: the barriers questionnaire II (BQ-II). Pain 2002, 99(3):385-396.

32. Scott A: Identifying and analysing dominant preferences in discrete choice experiments: an application in health care. J Econ Psychol 2002, 23:383-398.

33. Bryan S, Gold L: Preference measurement using conjoint methods: an empirical investigation of reliability. Health Econ 2000, 9:385-395.

\section{doi:10.1186/1472-6947-13-118}

Cite this article as: Meghani et al: Measuring preferences for analgesic treatment for cancer pain: How do African-Americans and Whites perform on choice-based conjoint (CBC) analysis experiments? BMC Medical Informatics and Decision Making 2013 13:118. 